\title{
Latent tuberculosis infection among contacts of patients with multidrug-resistant tuberculosis in New South Wales, Australia
}

\author{
Vicky Chang $\mathbb{C}^{1,2}$, Raphael Hongxi Ling ${ }^{1}$, Kavindhran Velen $\mathbb{1}^{1}$ and Greg J. Fox ${ }^{1,3}$
}

${ }^{1}$ Central Clinical School, The Faculty of Medicine and Health, The University of Sydney, NSW, Australia. ${ }^{2}$ The Sutherland Hospital, Caringbah, NSW, Australia. ${ }^{3}$ Royal Prince Alfred Hospital, Camperdown, NSW, Australia.

Corresponding author: Greg J. Fox (gregory.fox@sydney.edu.au)

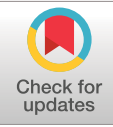

updates
Shareable abstract (@ERSpublications)

Given the high likelihood of multidrug resistance in strains responsible for LTBI among MDR-TB contacts, new research is needed to evaluate preventive therapies for this patient population https://bit.ly/2Tf4LLY

Cite this article as: Chang V, Ling $\mathrm{RH}$, Velen $\mathrm{K}$, et al. Latent tuberculosis infection among contacts of patients with multidrug-resistant tuberculosis in New South Wales, Australia. ERJ Open Res 2021; 7 : 00149-2021 [DOI: 10.1183/23120541.00149-2021].
Copyright (CThe authors 2021

This version is distributed under the terms of the Creative Commons Attribution NonCommercial Licence 4.0. For commercial reproduction rights and permissions contact permissions@ersnet.org

Received: 5 March 2021 Accepted: 21 June 2021

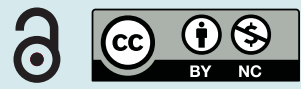

\section{Abstract}

Background Contacts of an individual with active tuberculosis (TB) disease have a higher risk of developing latent TB infection (LTBI) or active TB disease. Contact tracing is a public health measure that seeks to identify exposed contacts, screen them for co-prevalent TB and consider prophylactic treatment to prevent progression from LTBI to active TB disease. The investigators sought to determine the prevalence of LTBI and active TB disease among contacts of patients with multidrug-resistant (MDR)-TB in New South Wales, Australia.

Methodology A retrospective cohort study was performed among the contacts of patients diagnosed with MDR-TB between 2000 and 2016, inclusive, at seven chest clinics. Medical records were used to identify eligible contacts. Outcomes of screening and prophylactic treatment regimens offered to MDR-TB contacts with LTBI were characterised. Collected data included demographic information, screening tests results and initial management.

Results In total, 247 contacts of 55 MDR-TB patients were identified. LTBI was identified in 105 contacts (42.5\%). Preventive treatment was received by 20 contacts with LTBI $(32.3 \%)$ in the form of various regimens, ranging from one to three antimicrobials, with various doses and durations. One contact with LTBI who was untreated progressed to active TB disease during the study period, according to clinic notes. Conclusion Contacts of MDR-TB patients have a high prevalence of LTBI. Management of these contacts varies substantially in New South Wales, reflecting a lack of definitive evidence for preventive therapy. Further research is required to determine the optimal management of this population.

\section{Introduction}

In 2018, an estimated 10 million people fell ill with tuberculosis (TB) worldwide [1, 2]. Among these, almost half a million people developed multidrug-resistant (MDR)-TB, defined as disease caused by Mycobacterium tuberculosis that is resistant to both isoniazid and rifampicin [1-4]. A diagnosis of MDR-TB presents a major challenge for patients, owing to the prolonged treatment, high incidence of adverse events, high mortality rates and substantial costs of treatment $[5,6]$.

TB is transmitted via droplets that are coughed up by infected patients and inhaled by susceptible contacts. The World Health Organization (WHO) aims to eliminate this disease by 2035 [7]. In high-income countries with low rates of $\mathrm{TB}$, contact tracing is the primary method used to find those at risk of developing TB. However, the prevalence of latent tuberculosis infection (LTBI) and TB disease among contacts of patients with MDR-TB and drug-susceptible TB are not well understood. Systematic reviews and meta-analyses have shown that contacts of MDR-TB patients have a high risk of developing TB, with disease commonly diagnosed within 12 months of the index patient's diagnosis [8,9]. A recent prospective cohort study found a higher prevalence of LTBI among household contacts of MDR-TB patients than 
among contacts of patients with newly diagnosed TB that was presumed not to be drug resistant [10]. Accelerating the detection of disease among high-risk contacts of MDR-TB patients could maximise the opportunity to identify and treat latent infection to prevent disease, and should be prioritised to achieve the End TB Strategy [11].

Identifying MDR-TB contacts with LTBI is clinically useful only if there is an approach to reducing the risk of developing active TB disease. This is the rationale for prophylactic treatment regimens for LTBI [12]. However, established consensus among global authorities and governments has yet to be reached with regards to managing LTBI in MDR-TB contacts [13-15]. In the absence of definitive guidelines in New South Wales (NSW), Australia, the provision of prophylaxis lies with a respiratory physician's clinical judgment, based upon the individual clinical features of each patient. A contact tracing study conducted in Victoria, Australia, reported substantial variability in prescribed prophylaxis for MDR-TB contacts, in terms of antimicrobial regimens as well as treatment duration [16]. Current practices regarding effective regimens to prevent TB among contacts of patients with MDR-TB have not yet been established [17].

This study aimed to determine the prevalence of LTBI and active TB disease among contacts of patients with MDR-TB screened in NSW and to characterise the current approach to managing LTBI in this population.

Methodology

Study design and setting

This retrospective cohort study was performed for contacts of patients with MDR-TB in NSW between 2000 and 2016. MDR-TB was defined as bacteriologically confirmed TB with resistance to isoniazid and rifampicin. Patients were excluded if they did not have bacteriologically confirmed MDR-TB (with proven isoniazid and rifampicin resistance). We collected quantitative data from the medical records of contacts of the MDR-TB index patients attending chest clinics within NSW. Variables of interest included demographic information, age, gender, country of birth and contact exposure to a person with known TB (an "index" patient). We also recorded disease status of the contacts, and the outcomes of the contact screening. Tuberculin skin test (TST) and interferon- $\gamma$ release assay (IGRA) test results of the contacts were recorded. Immunological evidence of TB infection was defined as a TST reaction size of $\geqslant 10 \mathrm{~mm}$ or a positive IGRA, upon which LTBI status was determined and the use of prophylactic treatment regimens was reported. Contacts were defined as having completed screening if they completed a TST or IGRA and clinical follow-up at the TB clinic.

\section{Participant identification}

A list of all patients with a laboratory-confirmed diagnosis of MDR-TB in NSW from January 1, 2000, to December 31, 2016, was identified from the state-wide mycobacterial reference laboratory at the Institute for Clinical Pathology and Medical Research Mycobacterium Reference Laboratory. In total, 55 patients were identified from seven participating urban chest clinics in NSW where patients received treatment at the TB clinics. We visited the chest clinics of these seven hospitals to identify the records of these MDR-TB patients, which contained documentation of routine contact tracing performed for each case of active TB disease.

Contact tracing was managed differently across different chest clinics, with some keeping paper records and others using specialised computer software. Generally, for each MDR-TB patient, a list of contacts would be identified and an attempt made to screen these individuals with follow-up at the chest clinic. The sample of MDR-TB contacts examined by this study included all individuals identified through chest clinic contact tracing programmes and comprised the population of identifiable MDR-TB contacts. No identified contacts were excluded at the data collection stage. Existing electronic and hard copy contact records maintained by chest clinics were obtained and manually searched for relevant quantitative data.

\section{Outcome measures}

Outcomes of contact screening were also of interest. Contacts with positive TST results obtained from contact screening, without a prior history of LTBI or TB, were considered to have new LTBI. We also recorded the use of prophylactic treatment regimens, including the medications used and their doses, durations and documented adverse effects. Adverse events were evaluated based upon documentation by the treating physicians in the patients' medical records. The grade of adverse events was determined from the available clinical information by two researchers (VC and RL), according to standardised criteria [8]. Grade 1 adverse events comprised events with no symptoms or mild symptoms; Grade 2 events comprised adverse events for which local or noninvasive interventions were indicated; Grade 3 events were defined as those requiring hospitalisation or resulting in disability; Grade 4 events comprised events with 


\begin{tabular}{|c|c|}
\hline Total contacts commenced screening & $247(100)$ \\
\hline \multicolumn{2}{|l|}{ Gender } \\
\hline Male & $107(43.3)$ \\
\hline Female & $140(56.7)$ \\
\hline \multicolumn{2}{|l|}{ Age, years } \\
\hline$<18$ & $27(10.9)$ \\
\hline $18-24$ & $16(6.4)$ \\
\hline $25-34$ & $76(30.8)$ \\
\hline $35-44$ & $43(17.4)$ \\
\hline $45-54$ & $38(15.4)$ \\
\hline $55-64$ & $29(11.7)$ \\
\hline $65+$ & $18(7.3)$ \\
\hline \multicolumn{2}{|l|}{ Country of birth } \\
\hline Africa & $12(4.9)$ \\
\hline East Asia & $18(7.3)$ \\
\hline South Asia & $26(10.5)$ \\
\hline Southeast Asia & $65(26.3)$ \\
\hline West Asia & $4(1.6)$ \\
\hline Europe & $4(1.6)$ \\
\hline South America & $1(0.4)$ \\
\hline North America & $1(0.4)$ \\
\hline Australia and New Zealand & $81(32.8)$ \\
\hline Not documented & $35(14.2)$ \\
\hline \multicolumn{2}{|l|}{ Employment status } \\
\hline Working full-time & $87(35.2)$ \\
\hline Working part-time & $91(36.8)$ \\
\hline Unemployed & $23(9.3)$ \\
\hline Studying & $31(12.6)$ \\
\hline Not stated & $15(6.1)$ \\
\hline \multicolumn{2}{|l|}{ Smoking status } \\
\hline Lifelong non-smoker & $185(74.9)$ \\
\hline Current smoker & $12(4.9)$ \\
\hline Ex-smoker & $15(6.1)$ \\
\hline Not documented & $35(14.2)$ \\
\hline \multicolumn{2}{|l|}{ Alcohol usage } \\
\hline Does not drink & $159(64.4)$ \\
\hline Social usage & $58(23.5)$ \\
\hline Excessive usage & $8(3.2)$ \\
\hline Not documented & $22(30.9)$ \\
\hline \multicolumn{2}{|l|}{ IVDU } \\
\hline Non-IVDU & $222(89.9)$ \\
\hline Current IVDU & $0(0)$ \\
\hline Ex-IVDU & $3(1.2)$ \\
\hline Not documented & $22(30.9)$ \\
\hline \multicolumn{2}{|l|}{ Bacille Calmette-Guérin scar } \\
\hline Yes & $57(23.1)$ \\
\hline No & $30(12.1)$ \\
\hline Not documented & $160(64.8)$ \\
\hline \multicolumn{2}{|l|}{ Medical comorbidities } \\
\hline \multicolumn{2}{|l|}{ HIV } \\
\hline Yes & $0(0)$ \\
\hline No & $221(89.5)$ \\
\hline Not documented & $26(10.5)$ \\
\hline \multicolumn{2}{|l|}{ Diabetes mellitus } \\
\hline Yes & $30(12.1)$ \\
\hline No & $209(84.6)$ \\
\hline Not documented & $8(3.2)$ \\
\hline \multicolumn{2}{|l|}{ Immunosuppression } \\
\hline Yes & $0(0)$ \\
\hline No & $231(93.5)$ \\
\hline Not documented & $16(6.5)$ \\
\hline
\end{tabular}




\begin{tabular}{ll}
\hline TABLE 1 Continued & \\
\hline Types of contact & $94(38)$ \\
\hline Household & $71(28.7)$ \\
\hline Institutional/healthcare & $10(4)$ \\
\hline Other & $72(29.1)$ \\
\hline Not documented & \\
\hline Data presented as n (\%). MDR: multidrug resistant; TB: tuberculosis; IVDU: intravenous drug user. \\
\hline
\end{tabular}

life-threatening consequences; and Grade 5 events resulted in death. Adverse events leading to changes in treatment were documented.

\section{Data analysis}

Descriptive analyses were performed. Quantitative variables are summarised using frequency (\%) and median (interquartile range (IQR)). Adverse events were stratified by organ or system. Proportions were based upon the number of non-missing values. Statistical analyses were performed using IBM SPSS Statistics (version 25.0, IBM Corp., Armonk, NY, USA).

\section{Ethical considerations}

Ethical approval for the study was granted by the Sydney Local Health District Human Research Ethics Committee (LNR/17/CRGH/129). Site-specific permission was obtained from each hospital prior to commencement of data collection.

\section{Results}

We reviewed the medical files of 55 MDR-TB patients from seven chest clinics in Sydney, NSW, through which 247 MDR-TB contacts were screened (table 1). The number of contact cases per index patient ranged from one to 115 with a median of two contacts per case. Most of the contacts (131 of 247, 53.0\%) were born overseas. All patients with Bacille Calmette-Guérin (BCG) scars (57 of 247, 23.1\%) were born overseas.

247 contacts with chest clinic records commenced screening, of which 215 completed screening. TST was the predominant mode of TB exposure screening. Only three contacts were documented to have had an IGRA test. Immunological evidence of TB infection was found in 105 contacts (42.5\%). 96 (38.9\%) were diagnosed with LTBI by the specialist medical assessment. 62 contacts (25.1\%) were classified as having LTBI due to recent exposure (i.e. newly diagnosed) and 34 (13.8\%) were classified as having LTBI due to remote exposure. Of the 62 newly diagnosed LTBI contacts, 11 (4.5\%) were born in Australia/New Zealand, 28 (11.3\%) in Southeast Asia, 13 (5.3\%) in South Asia, eight (3.2\%) in East Asia and two $(0.8 \%)$ in Africa. One contact had co-prevalent extrapulmonary TB that was fully sensitive to first-line antibiotics (figure 1). The index case isolate had different drug susceptibility test results from this contact. Table 2 shows the screening outcomes of contacts.

More than half of the contacts with newly diagnosed LTBI were offered prophylactic treatment (table 3). Extensive variation was observed in the medications, doses and durations making up the prophylactic treatment regimens offered to contacts with LTBI. Table 4 reports the documented regimens used in the treating hospitals. In two contacts, isoniazid preventive therapy was commenced before patients' drug susceptibility results were known. Treating clinicians stopped therapy once it was recognised to be ineffective. These two contacts went onto chest X-ray surveillance. 14 of the index cases with MDR-TB had organisms that were susceptible to high-level isoniazid, and 11 contacts were offered isoniazid as part of their prophylactic regimens. Adverse events from the prophylactic treatments were rarely reported.

\section{Discussion}

This retrospective cohort study found a high prevalence of LTBI among contacts of patients diagnosed with MDR-TB over a 16-year period. In low-incidence countries, including Australia, that have low background transmission rates of $\mathrm{TB}$, transmission among contacts born in those countries most likely results from the recognised exposure to a patient with MDR-TB. The calculated LTBI prevalence in our study was 96 of 247 (38.9\%), which is comparable to a reported 52.6\% prevalence of LTBI in a meta-analysis of contacts of patients with MDR-TB or extensively drug-resistant TB in other high-income countries [8]. One contact had concurrent extrapulmonary TB that was not drug resistant. More than half 


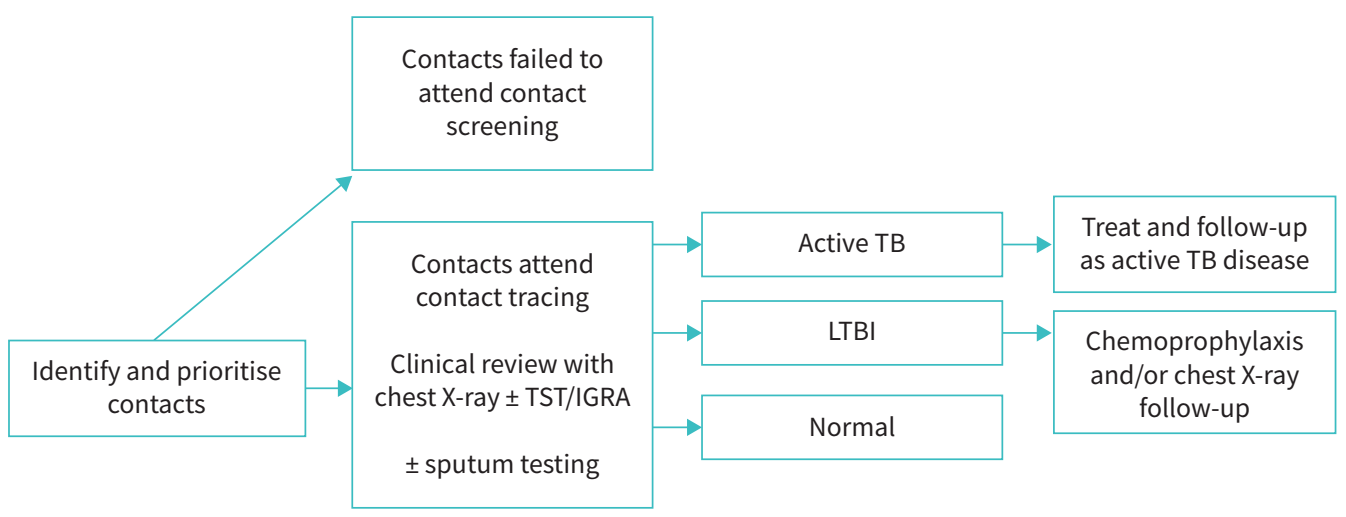

FIGURE 1 Algorithm for contact tracing and management in New South Wales, Australia. TST: tuberculin skin test; IGRA: interferon- $\gamma$ release assay; TB: tuberculosis; LTBI: latent TB infection.

of the contacts with LTBI (33 of 62, 53.2\%) were offered prophylactic treatment but only 20 of 62 (32.3\%) of the contacts accepted preventive therapies. The majority of the contacts (42 of 62, 67.7\%) accepted chest X-ray surveillance.

Selecting an appropriate preventive therapy is a major challenge in treating contacts of patients with drug-resistant TB. It is often difficult to distinguish whether contact LTBI arises from transmission from the recognised index case or from a prior remote infection. For those born in Australia, a setting with a low incidence and low background transmission of $M$. tuberculosis, most cases of LTBI are likely to have occurred owing to their close contact with active MDR-TB disease. However, in our study, many contacts were born overseas, meaning that it was difficult to definitively exclude remote infection.

This study had several limitations. First, because this was a retrospective study, documentation in the medical records was sometimes incomplete. By relying on chest clinic records, the investigators are unable to exclude the possibility that some contacts were not documented in available records. Second, with the small number of contacts treated, the variation in regimens used and the absence of a control group, we

TABLE 2 Screening outcomes of contacts of patients with MDR-TB, New South Wales, Australia, 2000-2016

$\begin{array}{lc}\text { Contacts who commenced screening } & 247(100) \\ \text { Completed screening (total) } & 215(87) \\ \text { Tested positive for LTBI } & 105(42.5) \\ \text { TST positive }(\geqslant 10 \mathrm{~mm}) & 104(42.1) \\ \text { IGRA positive }(\geqslant 0.35) & 1(0.4) \\ \text { Tested negative for LTBI } & 110(44.5) \\ \text { TST negative }(<10 \mathrm{~mm}) & 108(43.7) \\ \text { IGRA negative }(<0.35) & 2(0.8) \\ \text { Did not complete screening } & 32(13.0) \\ \text { Did not attend } & 26(10.5) \\ \text { Transferred out before completing screening } & 3(1.2) \\ \text { Deceased before completing screening } & 3(1.2) \\ \text { Chest X-ray findings } & \\ \text { Abnormal } & 8(3.2) \\ \text { Normal } & 207(83.8) \\ \text { Not documented } & 32(13.0) \\ \text { Final diagnosis } & \\ \text { LTBI } & 96(38.9) \\ \text { Newly diagnosed LTBI } & 62(25.1) \\ \text { Prior history of LTBI } & 34(13.8) \\ \text { Active TB disease } & 1(0.4) \\ \text { Previous inactive TB (treated or not treated) } & 8(3.2)\end{array}$

Data presented as $\mathrm{n}(\%)$. MDR: multidrug resistant; TB: tuberculosis; LTBI: latent tuberculosis infection; TST: tuberculin skin test; IGRA: interferon- $\gamma$ release assay. 


\section{TABLE 3 Management of contacts diagnosed with LTB}

Contacts

\begin{tabular}{lc}
\hline Contacts with newly diagnosed LTBI & $62(100)$ \\
Preventive therapy offered & $31(50)$ \\
\hline Accepted & $18(29)$ \\
$\quad$ Refused & $13(21.0)$ \\
Preventive therapy not offered & $31(50)$ \\
Chest X-ray surveillance offered & $31(50)^{\#}$ \\
$\quad$ Accepted" & $44(71.0)$ \\
Refused & 0 \\
\hline
\end{tabular}

Data presented as $\mathrm{n}(\%)$. LTBI: latent tuberculosis infection. *: isoniazid preventive therapy was commenced before patients' drug susceptibility results were known, stopped by clinicians once it was recognised to be ineffective, and contacts offered chest X-ray surveillance; ${ }^{q}$ : including contacts refusing preventive therapy.

were unable to assess the effectiveness of different regimens to treat LTBI. The undertaking of this study has so far corroborated the utility of contact tracing programmes demonstrated by the existing body of literature and has characterised a substantial degree of contact transmission in NSW. Even if contacts do not receive treatment, they can be monitored for incipient disease following their known exposure. This is likely to accelerate diagnosis of early disease and reduce potential ongoing transmission in the community.

This study has important policy implications. First, given the significant role of contact transmission in LTBI development, efforts to detect and treat contacts with MDR-TB infection play an important role in the control of TB in this low-prevalence setting. LTBI is a significant risk for developing infectious active TB disease. It is often stated that the lifetime risk of developing active TB after an index infection is 5$10 \%$; a recent study has shown the 1650 -day cumulative hazard is up to $11.5 \%$ [18]. Hence, identifying contacts and screening them for LTBI can effectively break the cycle of TB transmission. Prevention is especially important for those at risk of MDR-TB, given the difficulty of treating these cases once active MDR-TB disease is established. Second, identifying contacts with LTBI is clinically useful only if there is an approach to reducing the risk of development into active TB disease. Regular clinical and radiological surveillance accelerate diagnosis of early disease and reduce potential ongoing transmission in the community [1], but they are labour intensive and do not reduce the risk of potential development into active TB disease. This is the rationale behind prophylactic treatment regimens for LTBI; however, currently available guidelines for treating LTBI in MDR-TB contacts lack uniformity and are based upon limited evidence of effectiveness of the commonly used preventive therapies in this population [2, 19, 20]. Another important consideration unique to our population of MDR-TB contacts would be individualising

\section{TABLE 4 Antibiotic treatment of infected contacts of patients with MDR-TB}

\begin{tabular}{|c|c|c|c|}
\hline $\begin{array}{l}\text { Regimen recommended by } \\
\text { treating physicians }\end{array}$ & $\begin{array}{c}\text { Contacts commencing } \\
\text { treatment }\end{array}$ & $\begin{array}{l}\text { Contacts completing } \\
\text { recommended } \\
\text { treatment }\end{array}$ & $\begin{array}{c}\text { Contacts reporting } \\
\text { adverse events } \\
\text { during treatment }\end{array}$ \\
\hline Total & $18(100)$ & $18(100)$ & $1(5)$ \\
\hline \multicolumn{4}{|l|}{ One drug } \\
\hline \multicolumn{4}{|l|}{ Moxifloxacin } \\
\hline 6 months & $7(35)$ & $7(35)$ & $0(0)$ \\
\hline \multicolumn{4}{|l|}{ Two drugs } \\
\hline Isoniazid and rifampicin & $7(35)$ & $7(35)$ & $0(0)$ \\
\hline 4 months & $3(15)$ & $3(15)$ & $0(0)$ \\
\hline 6 months & $3(15)$ & $3(15)$ & $0(0)$ \\
\hline Unknown duration & $1(5)$ & $1(5)$ & $0(0)$ \\
\hline \multicolumn{4}{|l|}{ Isoniazid and pyrazinamide } \\
\hline 9 months & $2(10)$ & $2(10)$ & $1(5)$ \\
\hline \multicolumn{4}{|l|}{ Three drugs } \\
\hline $\begin{array}{l}\text { Isoniazid, rifampicin and } \\
\text { pyrazinamide }\end{array}$ & $2(10)$ & $2(10)$ & $0(0)$ \\
\hline 5 months & $1(5)$ & $1(5)$ & $0(0)$ \\
\hline 6 months & $1(5)$ & $1(5)$ & $0(0)$ \\
\hline
\end{tabular}


therapy based on the drug-resistance profile of the index patient. Given the high likelihood of MDR in strains responsible for LTBI among MDR-TB contacts, prophylactic treatment regimens should ideally be guided by drug susceptibility profiles of M. tuberculosis strains.

\section{Conclusion}

This study has characterised the current practice relating to contact investigation for MDR-TB in NSW, and has shown a high prevalence of LTBI in close contacts of patients with MDR-TB. Further research is required to shape clinical guidelines relating to the appropriate management of LTBI among MDR-TB contacts. Attention should be given to identifying strategies that enhance uptake of screening, as well as chemoprophylaxis to advance the ambitious goal of TB elimination [7].

Acknowledgements: We acknowledge the patients whose data were evaluated in this study.

Provenance: Submitted article, peer reviewed.

Conflict of interest: None declared.

Support statement: G.J. Fox is supported by an Australian NHMRC Career Development Fellowship (APP\#1148372).

\section{References}

1 World Health Organization. Global Tuberculosis Report 2019. Geneva, World Health Organization, 2019.

2 World Health Organization. Companion Handbook to the WHO Guidelines for the Programmatic Management of Drug-Resistant Tuberculosis. Geneva, World Health Organization, 2014.

3 Mitchison DA. The diagnosis and therapy of tuberculosis during the past 100 years. Am J Respir Crit Care Med 2005; 171: 699-706.

4 Seddon JA, Garcia-Prats AJ, Purchase SE, et al. Levofloxacin versus placebo for the prevention of tuberculosis disease in child contacts of multidrug-resistant tuberculosis: study protocol for a phase III cluster randomised controlled trial (TB-CHAMP). Trials 2018; 19: 693.

5 Bastos ML, Hussain $\mathrm{H}$, Weyer $\mathrm{K}$, et al. Treatment outcomes of patients with multidrug-resistant and extensively drug-resistant tuberculosis according to drug susceptibility testing to first- and second-line drugs: an individual patient data meta-analysis. Clin Infect Dis 2014; 59: 1364-1374.

6 Ahmad N, Ahuja SD, Akkerman OW, et al. Treatment correlates of successful outcomes in pulmonary multidrug-resistant tuberculosis: an individual patient data meta-analysis. Lancet 2018; 392: 821-834.

7 World Health Organization. WHO Report to the World Health Assembly on the End TB Strategy and Targets. Geneva, World Health Organization, 2014.

8 Fox GJ, Barry SE, Britton WJ, et al. Contact investigation for tuberculosis: a systematic review and meta-analysis. Eur Respir J 2013; 41: 140-156.

9 Shah NS YC, Heo M, Tolman AW, et al. Yield of contact investigations in households of patients with drug-resistant tuberculosis: systematic review and meta-analysis. Clin Infect Dis 2014; 58: 381-391.

10 Fox GJ, Anh NT, Nhung NV, et al. Latent tuberculous infection in household contacts of multidrug-resistant and newly diagnosed tuberculosis. Int J Tuberc Lung Dis 2017; 21: 297-302.

11 Reichler MR, Khan A, Sterling TR, et al. Risk and timing of tuberculosis among close contacts of persons with infectious tuberculosis. J Infect Dis 2018; 218: 1000-1008.

12 World Health Organization. Latent Tuberculosis Infection. Updated and Consolidated Guidelines for Programmatic Management. Geneva, World Health Organization, 2018. https://apps.who.int/iris/bitsctream/ handle/10665/260233/9789241550239-eng.pdf Date last accessed: August 27, 2021.

13 van der Werf MJ, Sandgren A, Manissero D. Management of contacts of multidrug-resistant tuberculosis patients in the European Union and European Economic Area. Int J Tuberc Lung Dis 2012; 16: 426.

14 Marks SM, Mase SR, Morris SB. Systematic review, meta-analysis, and cost-effectiveness of treatment of latent tuberculosis to reduce progression to multidrug-resistant tuberculosis. Clin Infect Dis 2017; 64: 1670-1677.

15 Migliori GB, Tiberi S, Zumla A, et al. MDR/XDR-TB management of patients and contacts: challenges facing the new decade. The 2020 clinical update by the Global Tuberculosis Network. Int J Infect Dis 2020; 92: S15-S25.

16 Denholm JT, Leslie DE, Jenkin GA, et al. Long-term follow-up of contacts exposed to multidrug-resistant tuberculosis in Victoria, Australia, 1995-2010. Int J Tuberc Lung Dis 2012; 16: 1320-1325.

17 Fox GJ, Schaaf HS, Chiappini E, et al. Preventing the spread of multidrug-resistant tuberculosis and protecting contacts of infectious cases. Clin Microbiol Infect 2017; 23: 147-153.

18 Trauer JM, Moyo N, Tay EL, et al. Risk of active tuberculosis in the five years following infection...15\%? Chest 2016; 149: 516-525. 
19 Rodriguez CA, Sasse S, Yuengling KA, et al. A systematic review of national policies for the management of persons exposed to tuberculosis. Int J Tuberc Lung Dis 2017; 21: 935-940.

20 van der Werf MJ, Langendam MW, Sandgren A, et al. Lack of evidence to support policy development for management of contacts of multidrug-resistant tuberculosis patients: two systematic reviews. Int J Tuberc Lung Dis 2012; 16: 288-296. 